\title{
The Tendencies and Timeline of the Solar Burst Type II Fragmented
}

\author{
Z. S. Hamidi ${ }^{1, \star}$, N. N. M. Shariff ${ }^{2}$, C. Monstein ${ }^{3}$ \\ ${ }^{1}$ School of Physics and Material Sciences, Faculty of Sciences, MARA University of Technology, \\ 40450, Shah Alam, Selangor, Malaysia \\ ${ }^{2}$ Academy of Contemporary Islamic Studies (ACIS), MARA University of Technology, \\ 40450, Shah Alam, Selangor, Malaysia \\ ${ }^{3}$ Institute of Astronomy, Wolfgang-Pauli-Strasse 27, Building HIT, Floor J, \\ $\mathrm{CH}-8093$ Zurich, Switzerland \\ *E-mail address: zetysh@salam.uitm.edu.my
}

\begin{abstract}
We report the timeline of the solar radio burst Type II that formed but fragmented at certain point based on the eruption of the solar flare on $13^{\text {th }}$ November 2012 at 2:04:20 UT. The active region AR 1613 is one of the most active region in 2012. It is well known that the magnetic energy in the solar corona is explosively released before converted into the thermal and kinetic energy in solar flares. In this work, the Compound Astronomical Low-frequency, Low-cost Instrument for Spectroscopy Transportable Observatories (CALLIISTO) system is used in obtaining a dynamic spectrum of solar radio burst data. There are eight active regions and this is the indicator that the Sun is currently active. Most the active regions radiate a Beta radiation. The active regions 1610, 1611 and 1614 are currently the largest sunspots on the visible solar disk. There is an increasing chance for an isolated M-Class solar flare event. It is also expected that there will be a chance of an $\mathrm{M}$ flare, especially from AR 1614 and 1610. Although these two observations (radio and X-rays) seem to be dominant on the observational analysis, we could not directly confirmed that this is the only possibility, and we need to consider other processes to explain in detailed the injection, energy loss and the mechanism of the acceleration of the particles. In conclusion, the percentage of energy of solar flare becomes more dominant rather than the acceleration of particles through the Coronal Mass Ejections (CMEs) and that will be the main reason why does the harmonic structure of type II burst is not formed. This event is one fine example of tendencies solar radio burst type III, which makes the harmonic structure of solar radio burst type II fragmented.
\end{abstract}

Keywords: Sun; low frequency; solar radio; burst, type II; type III; e-CALLISTO

\section{INTRODUCTION}

It is well known that the magnetic energy in the solar corona is explosively released before converted into the thermal and kinetic energy in solar flares. 
The temperature of the explosion could exceed up to 10-20 MK. The acceleration of these particles can be described by parallel electric fields, drift velocities caused by perpendicular forces which is (E x B drifts) while gyromotion caused by Lorentz forces of the magnetic field (Aswaden 2006, Book). It is widely accepted that the solar radio burst type III plays a fundamental role in solar burst studies [1].

This burst classified as a short, strong burst which move rapidly from around $500 \mathrm{MHz}$ to lower frequencies and eject high energy electrons away from the sun at about $1 / 4$ the speed of light.

Meanwhile, type II radio bursts are caused by electrons accelerated in magnetohydrodynamic shocks driven by the solar eruptions [2]. However, how exactly these electrons are accelerated during solar eruptions is not fully understood, and is one of the outstanding issues of solar physics.

This type can be divided into two main categories which is (i) herring bones and (ii) harmonic structure pattern.in harmonic structure, it can be interpreted as the fundamental (F) and the second harmonic $(\mathrm{H})$ emissions generated via a plasma radiation mechanism at frequencies determined by the local plasma density (Ginzburg \& Zhelezniakov 1958). The onset time of this type precludes the possibility of the CME driven shock causing it [3].

The motion of the shock through the radial plasma density profile can be observed based on the decreasing of the signal in frequency. Solar radio burst type II were first identified by Payne Scott [4] and also discovered by Boischot [5] and classified as a broadband, lasting from 20 minutes to a few hours.

The latest interpretation of the type II burst emission is as follows: electrons accelerated in the Magnetohydrodynamics (MHD) shock front generate plasma waves, which get converted into electromagnetic radiation at the fundamental and harmonic of the local plasma frequency.

Type III solar burst, a fast drift burst is the most common of the meter wavelength bursts. The Type III solar burst was first introduced by Wild in 1963 [6] in the frequency range 500-10 MHz. It can be considered as a pre-flare stage that could be a signature of electron acceleration [7]. This type is related to solar flares and typically occurs before optical events. Further evidence presented that type III are generated in a weak-field region comes from the absence or low degree of circular polarization of the bursts.

The subject of nonlinear wave-wave interaction which involving interaction of electrostatic electron plasma that called as Langmuir waves active region radio emissions also have been studied [10-14].

The Langmuir waves originate from the nonthermal electrons, and the intensity of the radio bursts depends on the nonthermal electron density and energy. It is believed that a beam-plasma system is unstable to the generation of Langmuir waves, which are high frequency plasma waves at the local plasma frequency.

\section{EXPERIMENTAL SETUP AND OBSERVATION}

Modern antenna systems allow determination of the direction of the source of the solar burst radiation.The Compound Astronomical Low-frequency, Low-cost Instrument for Spectroscopy Transportable Observatories (CALLIISTO) system is used in obtaining a dynamic spectrum of solar radio burst data.

We have constructed the Log Periodic Dipole Antenna (LPDA) and this system was mounted on the top of the rooftop of National Space Centre (ANGKASA) building at Sg. 
Lang, Banting, Selangor located at ( $\left.\mathrm{N} 02^{\circ} 49.488^{\prime} \mathrm{E} 101^{\circ} 36.168^{\prime}\right)$. This antenna covered from 45-870 MHz [16].

This antenna is connected to the CALLISTO spectrometer via cable RG 58 and the modification, calibration process and basic testing of the antenna has been done in order to improve the quality of the system [17-21,26-35].

A preamplifier also is used to maximize the gain of the signal and all the data are automatically saved in FIT files. However, to avoid the interference signal, we focused the range of $150 \mathrm{MHz}$ till $350 \mathrm{MHz}$ [22,23].

This region is the best region with minimum interference at our site. We have selected the data from $220-380 \mathrm{MHz}$ region seems this is the best range with a very minimum of Radio Frequency Interference (RFI) [25].

\section{RESULTS AND ANALYSIS}

Based on the observation, a fast drift Type III solar burst is formed intermittently within six hours period of observation starting from 00:00 UT TO 3:00 UT. The chronology of the event is very important to understand what factors that caused the solar burst type II broke. This data is taken from our site and there are a few sites that also successfully obtained the data. Figure 1-11 shows the timeline of the solar burst type II and III.

It can be observed that solar radio burst type III in a group and individually are continue exploded within the first two hours. Sometimes the signal is so strong and sometimes is not. This shows a dynamic structure of the burst. The burst is origin from active region AR 1613 . We can actually observe a fast drift burst from $45 \mathrm{MHz}$ to $870 \mathrm{MHz}$.

The The results of the recent time indicate that the broken solar burst type II is formed within four minutes. Interestingly, in this case, type III solar burst can be observed before the formation type II burst. This is an indicator that the fast drift makes type II burst cannot form the harmonic structure.

The long fast-drift type Type III solar burst was recorded before the formation Type II solar burst. It is believed that the event is due to coherent plasma processes, so the frequency of emission is closely related to the plasma frequency (and hence the plasma density) of the medium through which the electrons propagate.

This observation allows for the mechanisms of evolution type II solar burst and local environment of the burst to be characterized. This event occurred from 2:00:03 UT till 2:00:06 UT. It peaked with M2.0 solar flare at $2.04 \mathrm{UT}$ in the range of $240-320 \mathrm{MHz}$. The burst that was temporally coincident with the X-ray region due to the M6.0 solar flare are are clearly seen. The type II burst started about two minutes later when the X-ray flux already declined to the background level. Previously, at 2:04:15 UT, solar radio burst Type III burst can be identified.

The type II burst is supposedly to be formed a harmonic structure. However, in this case, we could observe only a fundamental structure. In this case the role of type III shows the tendencies that type II burst could not form a harmonic structure during a very tense of type III burst.

Our next analysis will focus in the active region at the surface of the Sun. Figure 12 shows the position of Active Region 1613 and other active region that possible to create the solar active due to the eruption process. 


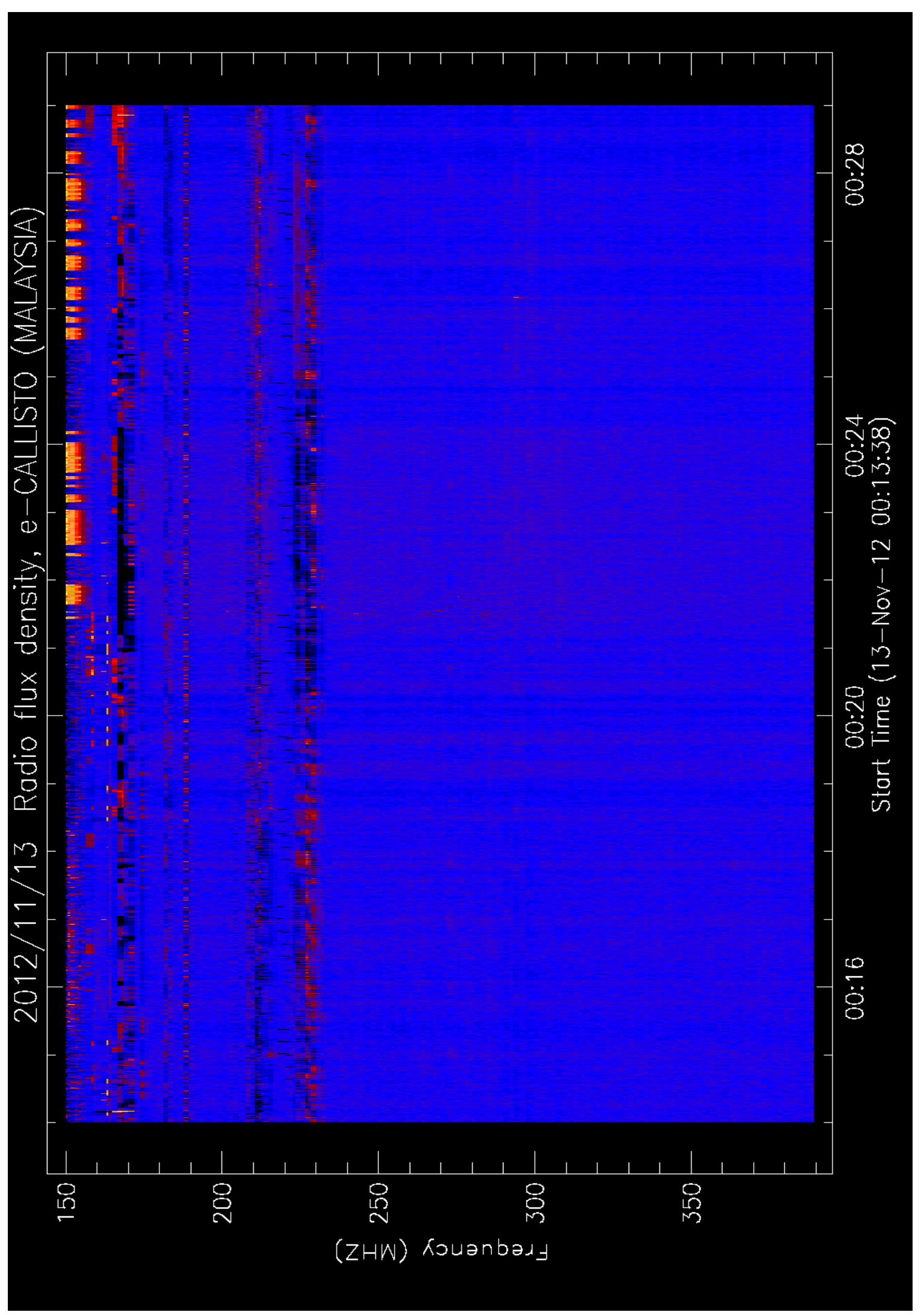

Figure 1. An intermittently and a group of type III burst from 00:00 - 00:30 UT. 


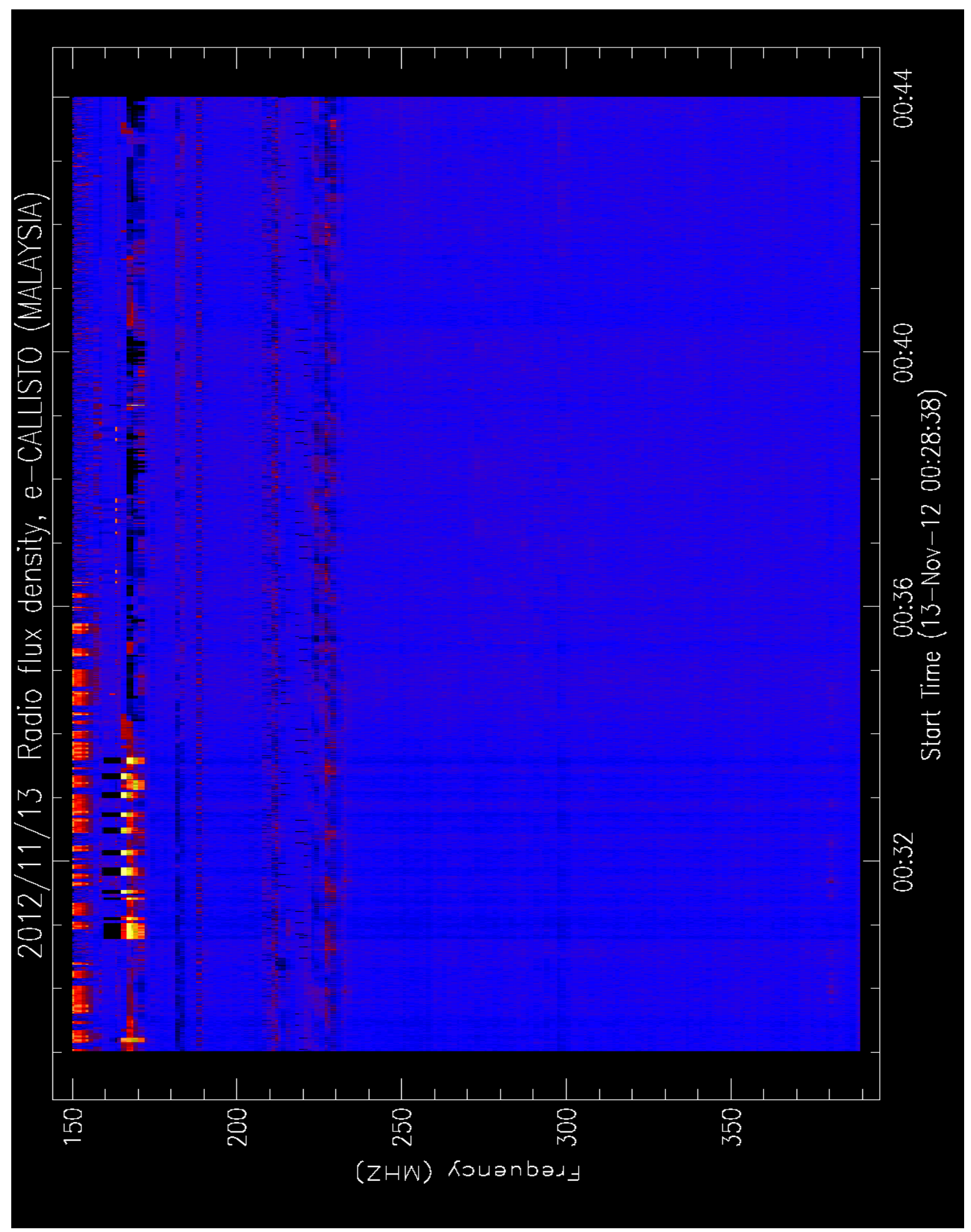

Figure 2. An intermittently and a group of type III burst from 00:30 - 00:45 UT. 


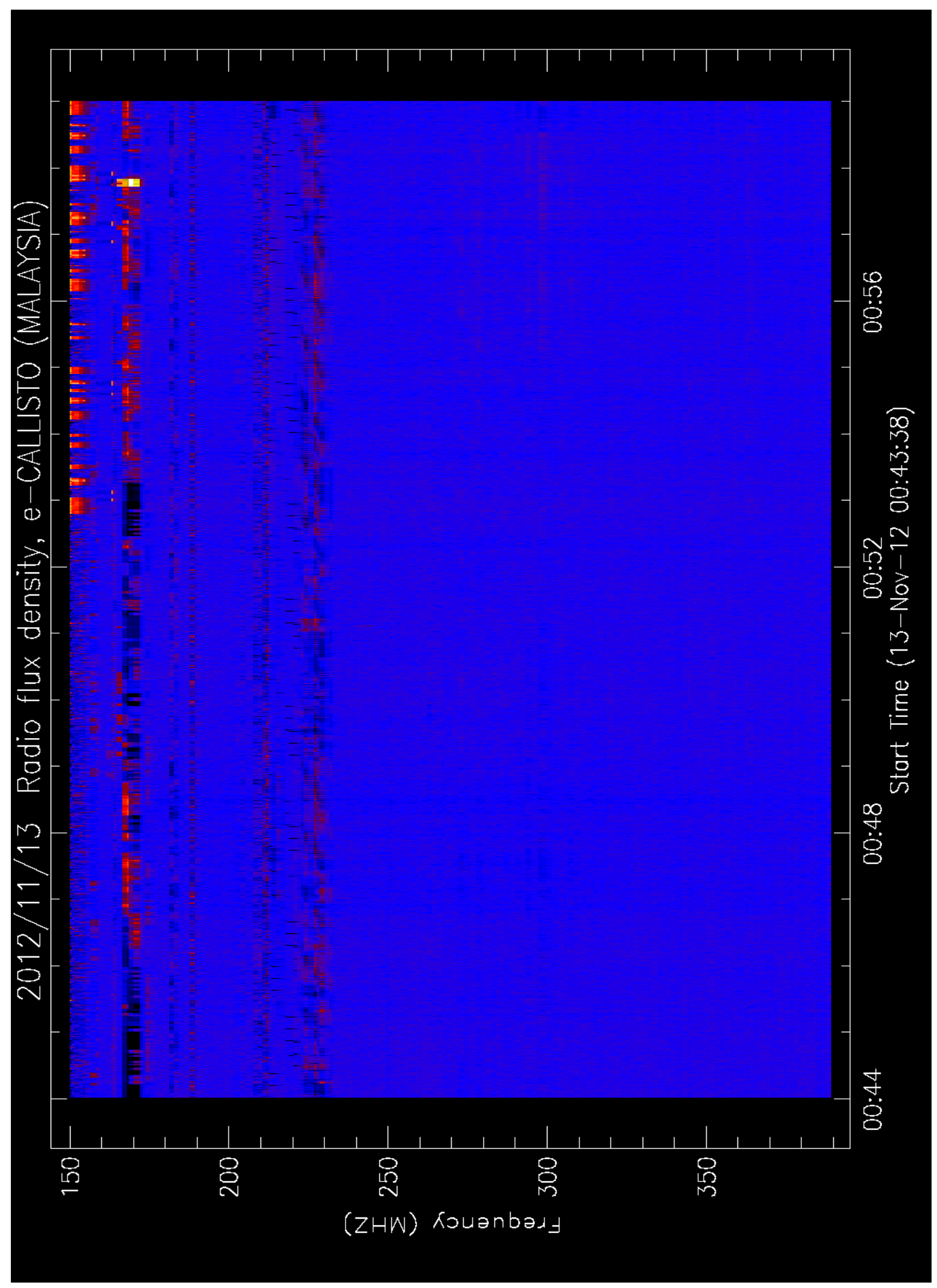

Figure 3. An intermittently and a group of type III burst from 00:45 - 01:00 UT. 


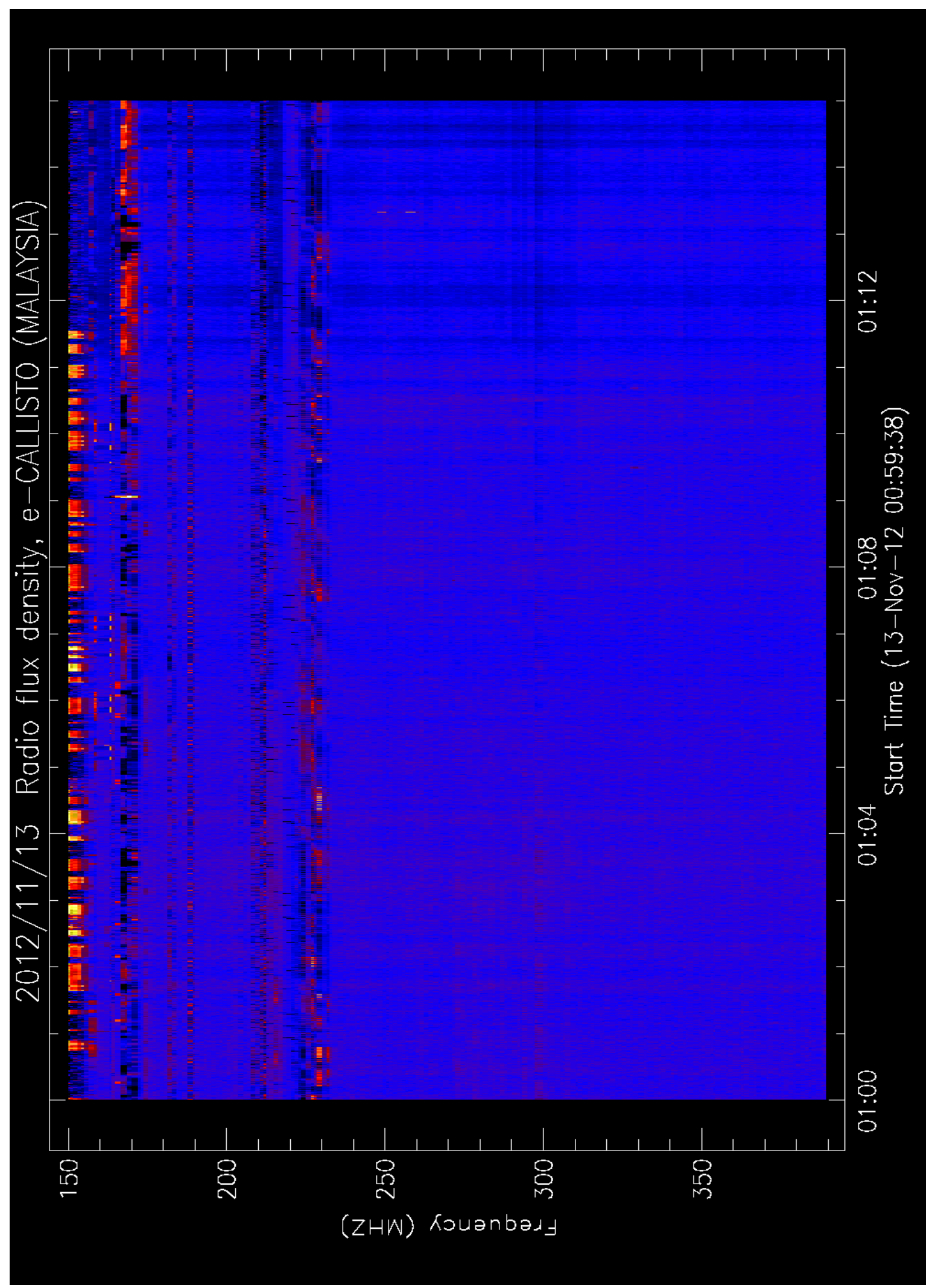

Figure 4. An intermittently and a group of type III burst from 01:00 - 01:15 UT. 


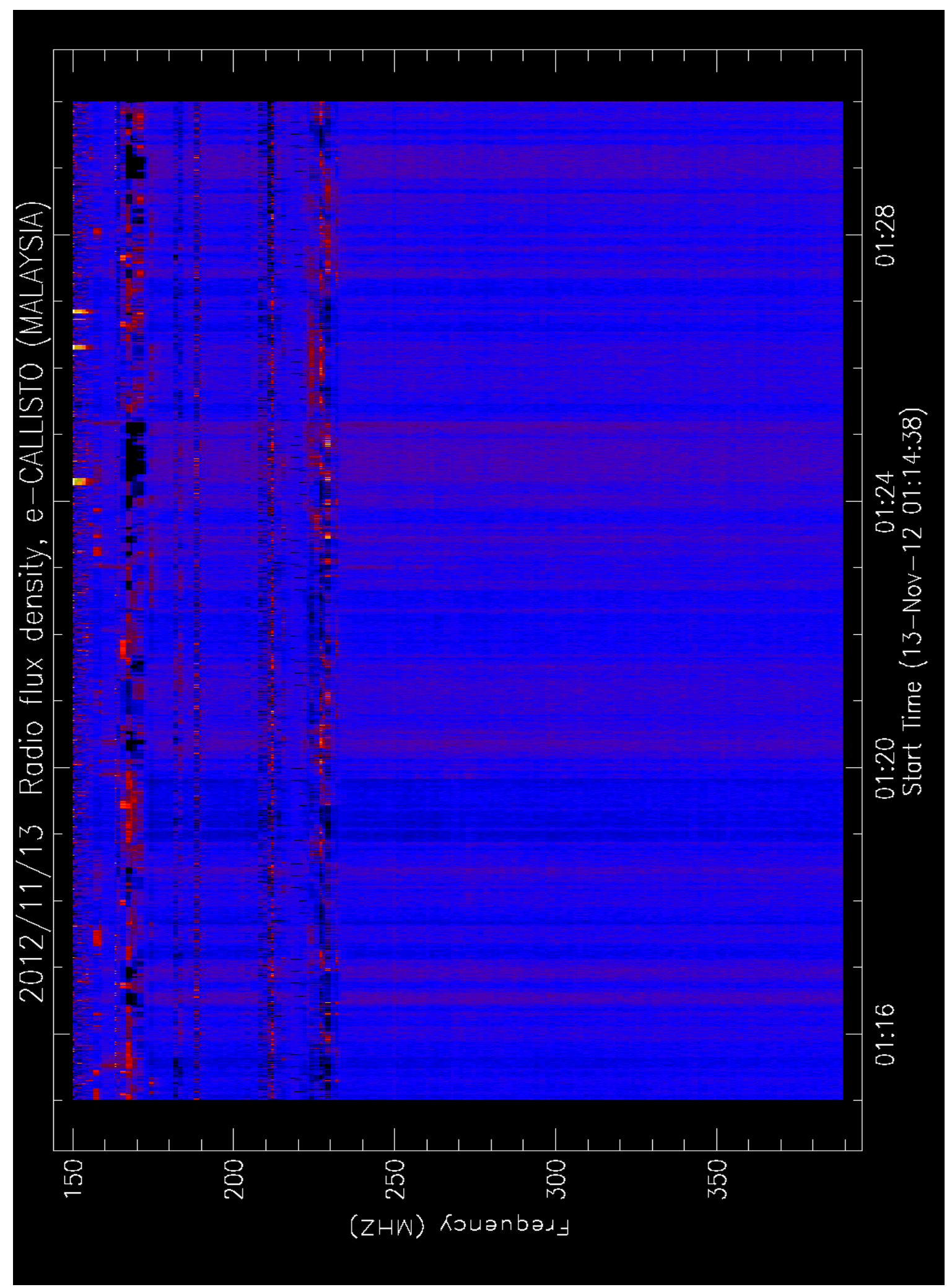

Figure 5. An intermittently and a group of type III burst from 01:15 - 01:30 UT. 


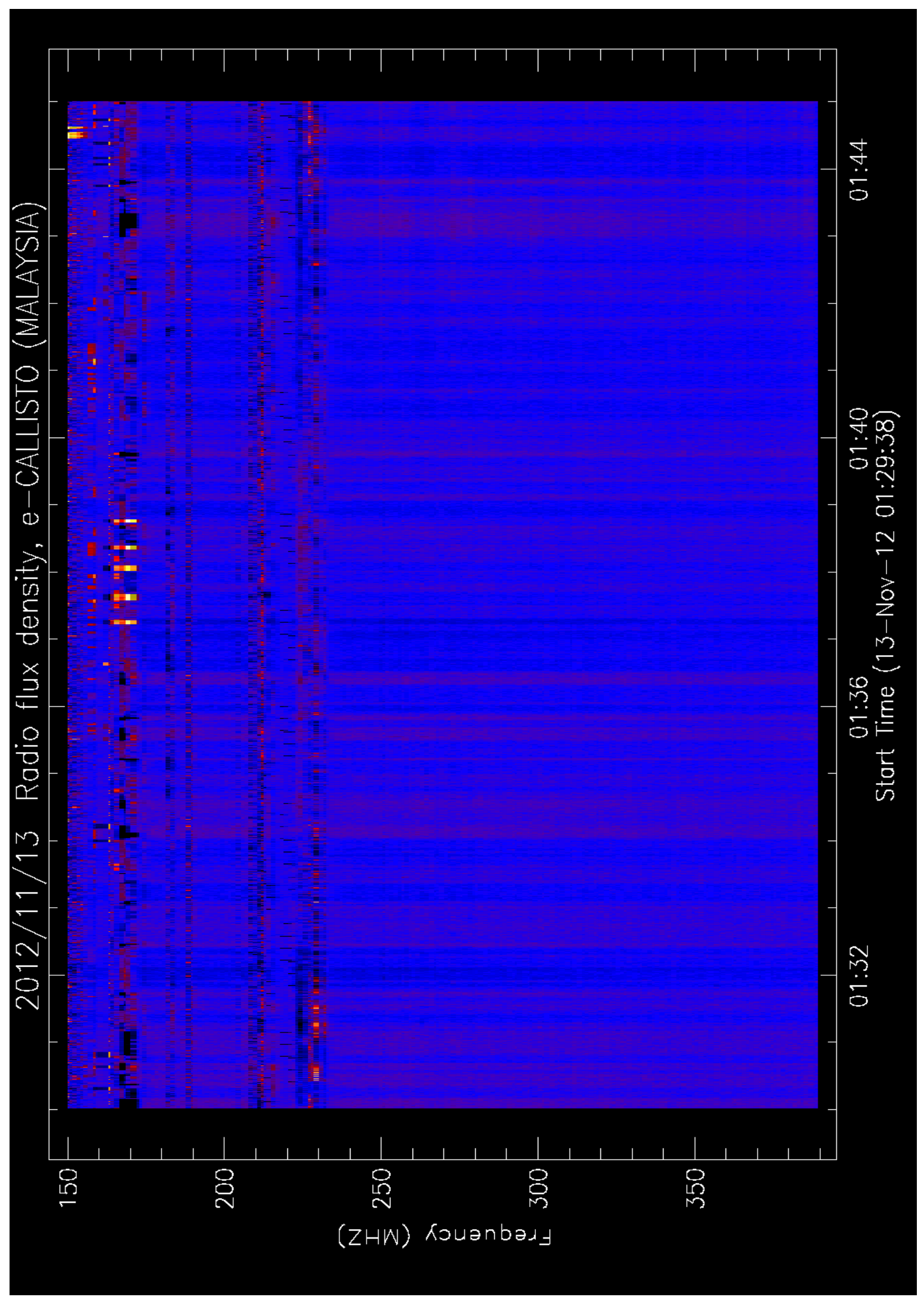

Figure 6. An intermittently and a group of type III burst from 01:30 - 01:45 UT. 


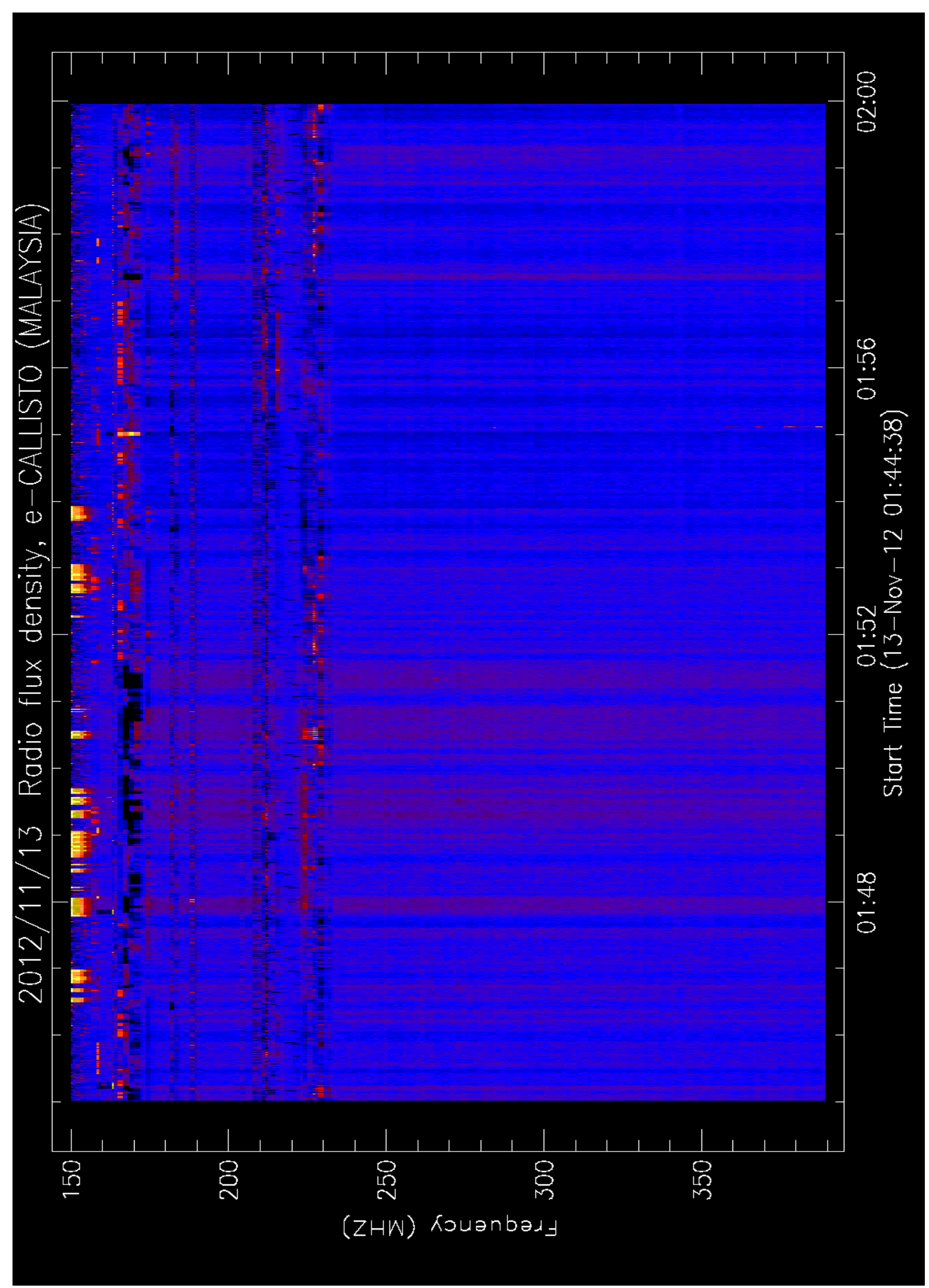

Figure 7. An intermittently and a group of type III burst from 01:45 - 02:00 UT. 


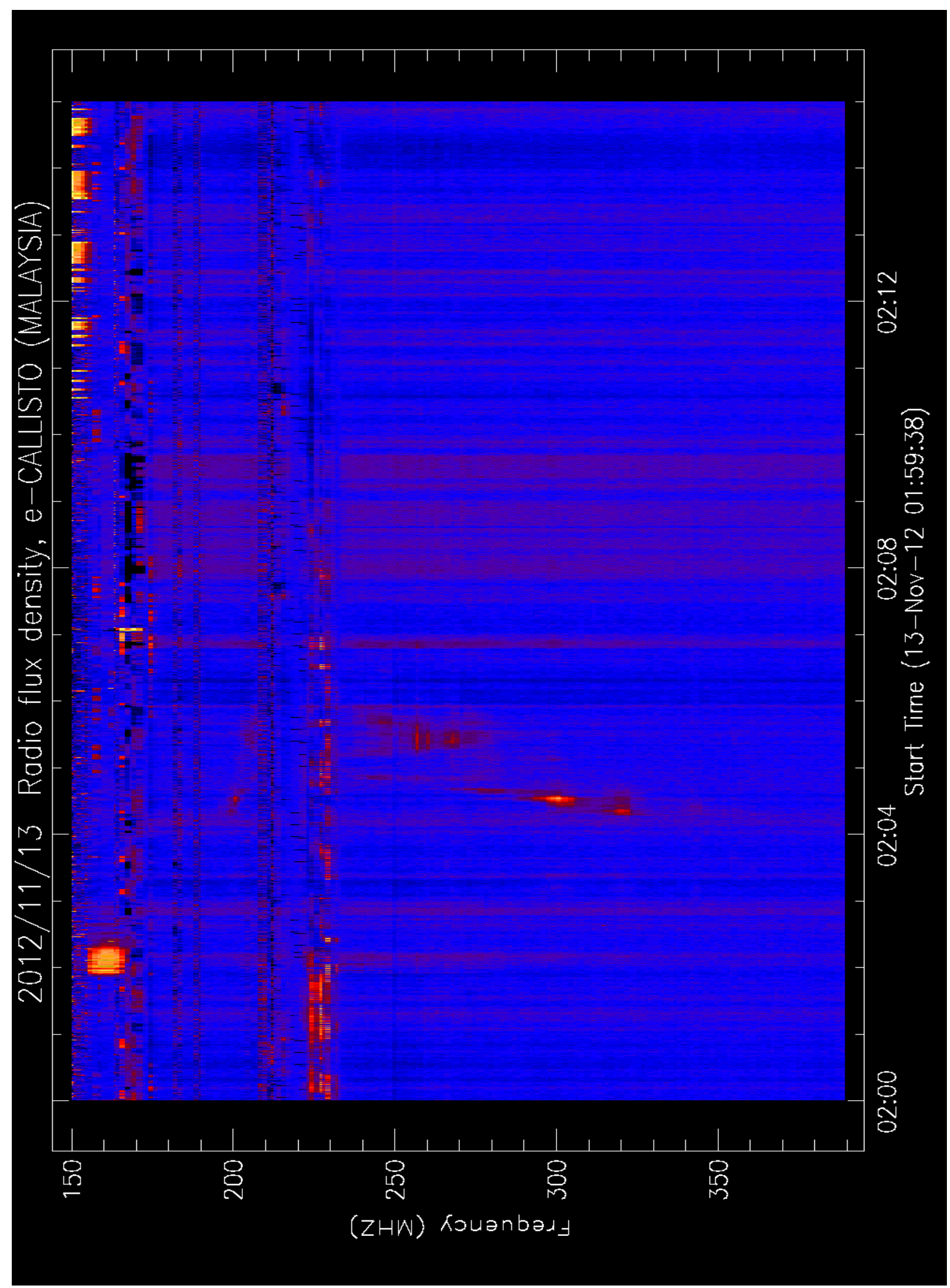

Figure 8. A fast drift type III, a broken type II burst and an intermittently and a group of type III burst from 02:00 - 02:15 UT. 


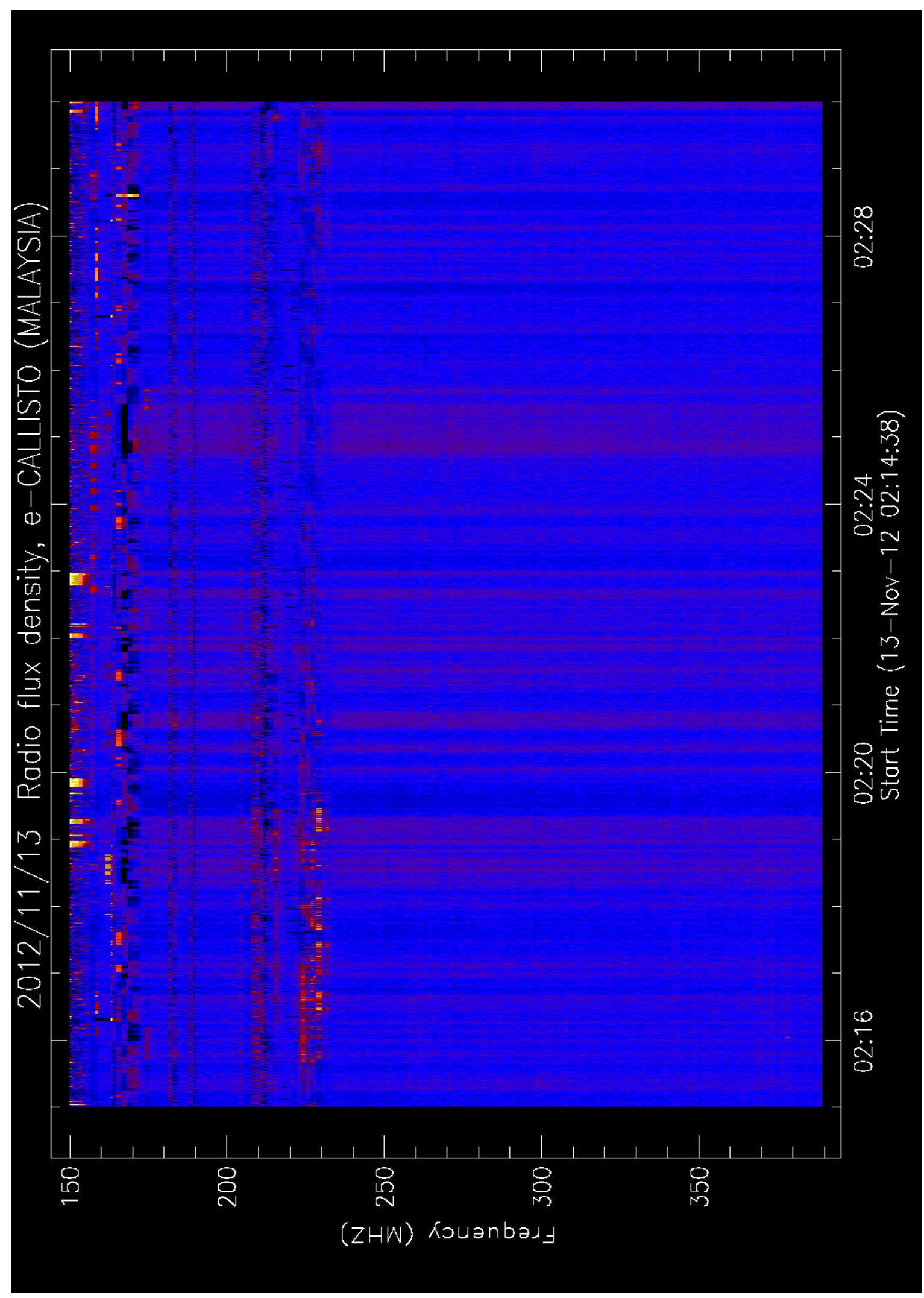

Figure 9. An intermittently and a group of type III burst from 02:15 - 02:30 UT. 


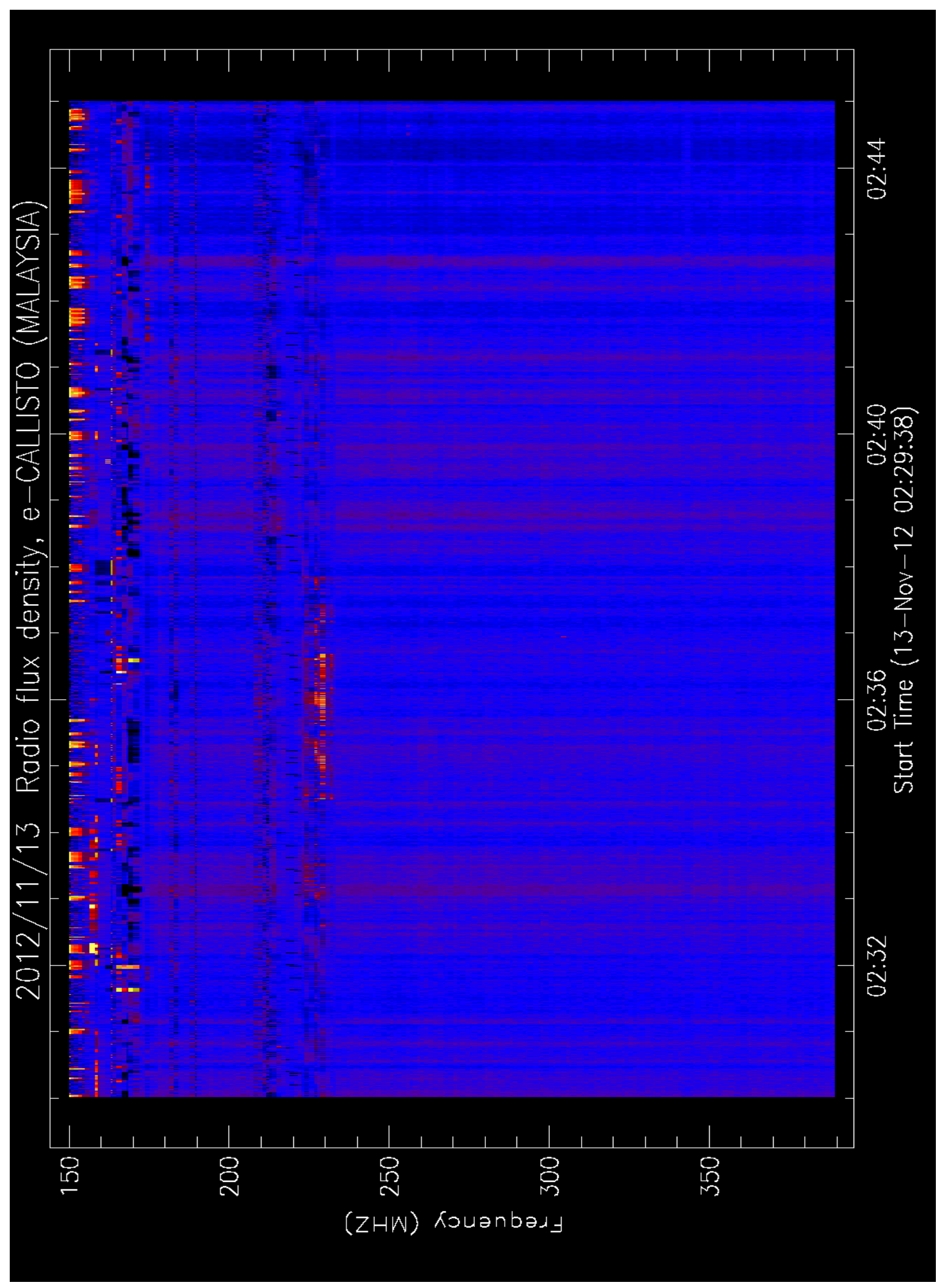

Figure 10. An intermittetly and a roup of type III burst from 02:30 - 02:45 UT. 


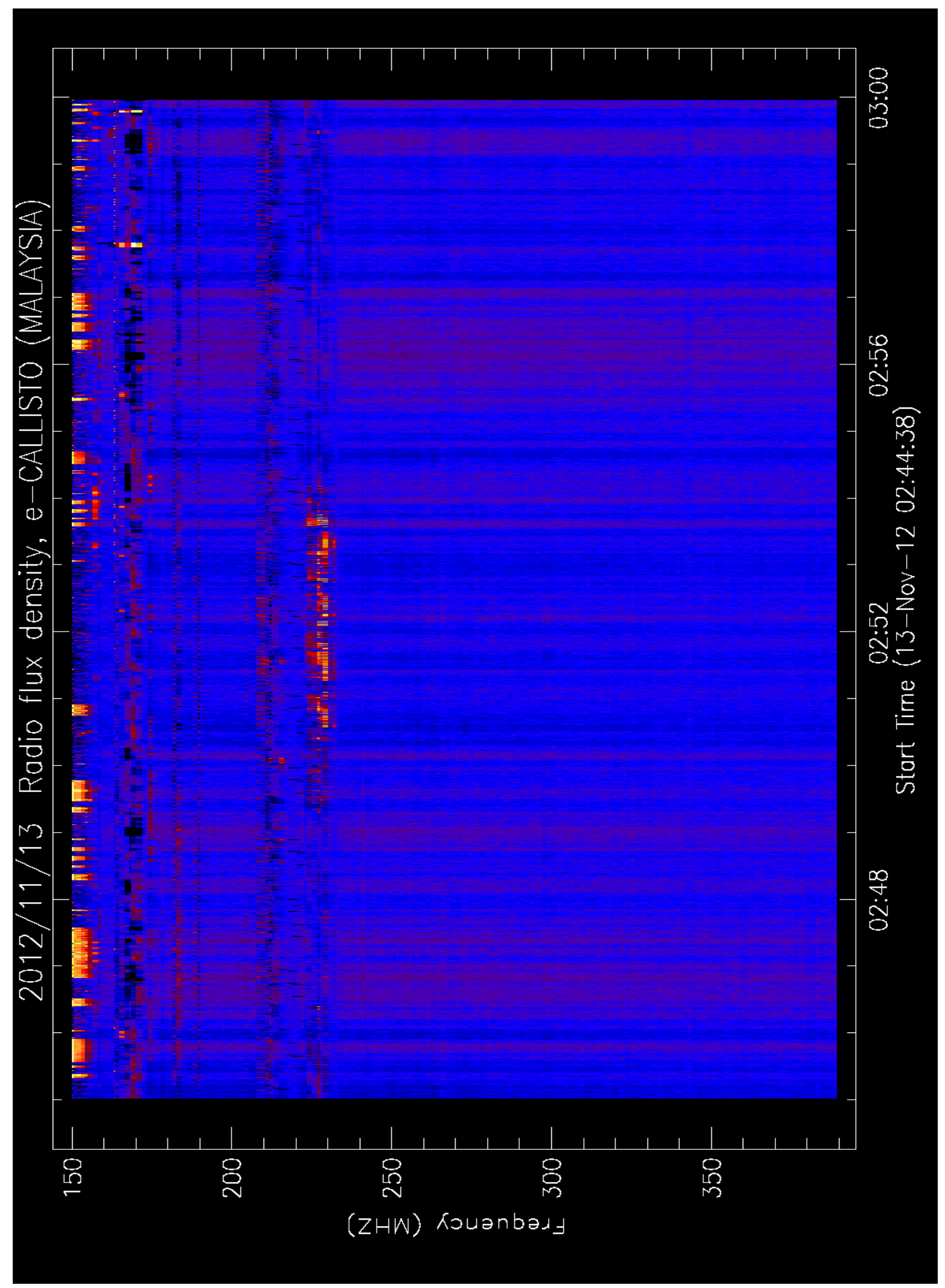

Figure 11. An intermittently and a group of type III burst from 02:45 - 03:00 UT. 


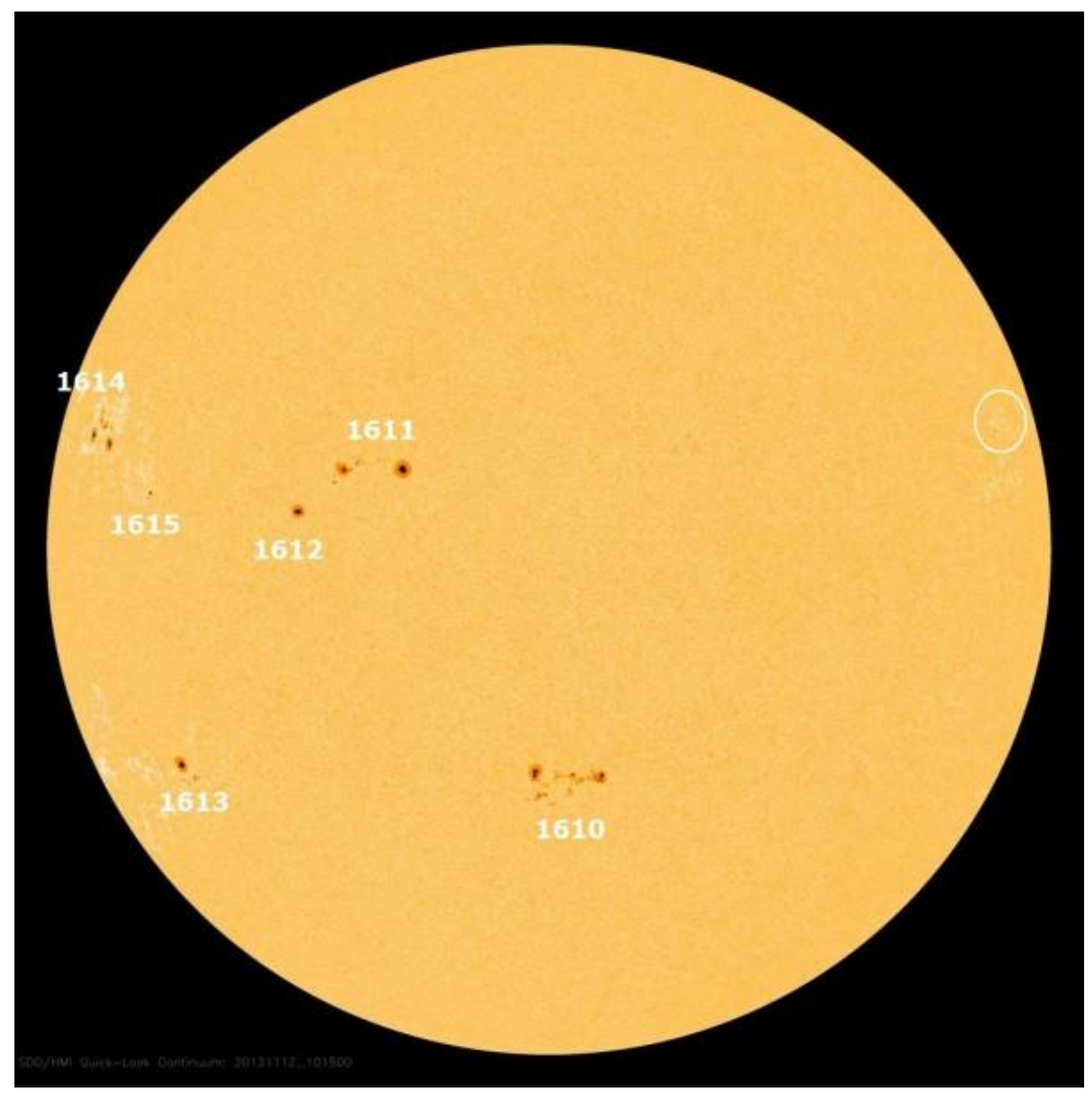

Figure 12. The position of Active Region 1613 (Credited to: Solar Dynamics Observatory (SDO).

The position of Active Region AR 1613 is illustrated in Figure 12. In order to get a clear picture about this unique event, we also make use the data from the Solar Dynamics Observatory (SDO) satellite. This instrument has unprecedented high cadences and sensitivities with $0-1.5 \mathrm{R} \odot$ and 12 seconds. There are a few sunspots that can be observed clearly and the Sun can be classified as an active Sun during this period. Solar activity has been eruptive during the past 24 hours, featuring three $\mathrm{C}$ flares and now fresh M-class flare.

The active regions 1610, 1611 and 1614 are currently the largest sunspots on the visible solar disk. There is an increasing chance for an isolated M-Class solar flare event. It is also expected that there will be a chance of an M flare, especially from AR 1614 and 1610.

Table 1 displays the detailed parameter of each active region that can be observed directly from ground and space observation during $13^{\text {th }}$ November 2012 . There are eight active regions and this is the indicator that the Sun is currently active. Some of the active region also remains exploded a huge particle and potentially eject the solar flares. Most the 
active regions radiate a Beta radiation. The location of the active region can be found in the North region of the Sun. Fortunately, the active region of 1613 is at the S $22^{\circ} \mathrm{E} 43^{\circ}$ and therefore, the explosion is not directly to the Earth.

Table 1. Parameter of each active region during $13^{\text {th }}$ November 2012.

\begin{tabular}{|c|c|c|c|c|c|c|c|}
\hline $\begin{array}{c}\text { Active } \\
\text { Region } \\
\text { Number }\end{array}$ & Location & Lo & Area & Z & LL & NN & Magnitude \\
\hline 1605 & N16W82 & 340 & 0010 & Bxo & 07 & 04 & Beta \\
\hline 1609 & S14W10 & 263 & 0010 & Hrx & 04 & 03 & Alpha \\
\hline 1610 & S22W10 & 263 & 0550 & Eki & 11 & 51 & Beta-Gamma \\
\hline 1611 & N15E13 & 240 & 0250 & Eho & 12 & 12 & Beta-Gamma \\
\hline 1612 & N09E25 & 228 & 0100 & Hsx & 05 & 01 & Alpha \\
\hline 1613 & S22E43 & 210 & 0170 & Dso & 10 & 10 & Beta \\
\hline 1614 & N16E58 & 195 & 0350 & Fho & 17 & 19 & Beta \\
\hline 1615 & N10E48 & 205 & 0160 & Cao & 10 & 08 & Beta \\
\hline
\end{tabular}

Table 2. The current condition of the Sun (Credited to Space Weather).

\begin{tabular}{|c|c|}
\hline Parameter & Value \\
\hline Solar wind speed & $369.7 \mathrm{~km} / \mathrm{sec}$ \\
\hline Density & $9.1 \mathrm{protons} / \mathrm{cm}^{3}$ \\
\hline Sunspot number & 188 \\
\hline $10.7 \mathrm{~cm}$ flux & $144 \mathrm{sfu}$ \\
\hline $6-\mathrm{hr}$ max & $\mathrm{M} 2$ \\
\hline $24-\mathrm{hr}$ & $\mathrm{M} 6$ \\
\hline
\end{tabular}

Overall, the Sun tends to be consistent with multiple storms, but not huge CMEs explosion. Based on LASCO observation, the wind explosion with a speed of $369.7 \mathrm{kms}^{-1}$. In conclusion, this event is one fine example of tendencies solar radio burst type III, which makes the harmonic structure of solar radio burst type II fragmented. 


\section{CONCLUDING REMARKS}

Although these two observations (radio and X-rays) seem to be dominant on the observational analysis, we could not directly confirmed that this is the only possibility, and we need to consider other processes to explain in detailed the injection, energy loss and the mechanism of the acceleration of the particles. Indirectly, it is believed that the large solar flares with a few numbers of solar storms contribute the distribution of flux energy or the burst. This energy of solar storms comes from the solar magnetic field which is generated from the convection zone. In conclusion, the percentage of energy of solar flare becomes more dominant rather than the acceleration of particles through the Coronal Mass Ejections (CMEs) and that will be the main reason why does the harmonic structure of type II burst is not formed.

\section{ACKNOWLEDGEMENT}

We are grateful to CALLISTO network, STEREO, LASCO,SDO/AIA, NOAA and SWPC make their data available online. This work was partially supported by the FRGS (600 RMI/FRGS 5/3 2012) UiTM grants. Special thanks to the National Space Agency and the National Space Centre for giving us a site to set up this project and support this project. Solar burst monitoring is a project of cooperation between the Institute of Astronomy, ETH Zurich, and FHNW Windisch, Switzerland, MARA University of Technology and University of Malaya. This paper also used NOAA Space Weather Prediction Centre (SWPC) for the sunspot, radio flux and solar flare data for comparison purpose. The research has made use of the National Space Centre Facility and a part of an initiative of the International Space Weather Initiative (ISWI) program.

\section{BIOGRAPHY}

Dr Zety Sharizat Hamidi is currently a lecturer and focused in Solar Astrophysics research specifically in radio astrophysics at the School of Physics and Material Sciences, Faculty of Sciences, MARA University of Technology, 40450, Shah Alam, Selangor, Malaysia. Involve a project under the International Space Weather Initiative (ISWI) and also a lecturer in School of Physics and Material Science, at MARA University of Technology, Shah Alam Selangor.

C. Monstein is a senior Engineer at Institute of Astronomy, Wolfgang-Pauli-Strasse 27, Building HIT, Floor J, CH-8093 Zurich, Switzerland and one of the researchers who initiated the CALLISTO system around the world.

Dr Nur Nafhatun Md Shariff is a senior lecturer in Academy of Contemporary Islamic Studies (ACIS), MARA University of Technology, 40450, Shah Alam, Selangor, Malaysia.Her current research is more on sustainability; environmental aspect. She is looking forward for cross-field research, i.e. solar astrophysics, light pollution measurement (mapping) and religious studies.

\section{References}

[1] M. R. Kundu, Solar Radio Astronomy, John Wiley, 1965.

[2] N. Gopalswamy, P. Makela, H. Xie, S.a.Y. Akiyama, S., CME interactions with coronal holes and their interplanetary consequences, J. Geophys. Res. (2009a).

[3] N. Gopalswamy, N. Nitta, P. K. Manoharan, A. Raoult, M. Pick, Astronomy and Astrophysics 347 (1999) 684-695.

[4] R. Payne-Scott, D. E. Yabsley, J. G. Bolton, Nature 160 (1947) 256-257.

[5] Boischot A., C. R. Acad. Sci. 244 (1957) 1326. 
[6] J. P. Wild, Smerd S. F., Weiss, A. A., Solar Burst, Ann. Rev. Astron. Astrophysics 1 (1963) 291-366.

[7] G. A. Dulk, Type III solar radio bursts at long wavelengths, in: R. Stone, E. Weiler, M. Goldstein (Eds.), Geophys. Monogr., 2000.

[8] A. F. Kuckes, Sudan R. N., Sol. Phys. 17 (1971).

[9] K. Kai, Sol. Phys. 11 (1970).

[10] G. B. a. L. Gelfreikh, B. I. , Soviet Astron. 23 (1979).

[11] P. Lantos, Sol. Phys. 22 (1972).

[12] A. Vourlidas, Bastian T. S., Nitta N., Aschwanden M. J., Sol. Phys. 163 (1996).

[13] E. Y. Zlotnik, Soviet Astron. 12 (1968).

[14] V.V. Zheleznyakov, Radio Emission of the Sun and Planets (1970).

[15] D. E. K. Gary, C. U., Solar and Space Weather Radiophysics Current Status and Future Developments Dordrecht: Kluwer, 2004.

[16] Z. S. Hamidi, Z. Ibrahim, Z. Abidin, M. Maulud, N. Radzin, N. Hamzan, N. Anim, N. Shariff, International Journal of Applied Physics and Mathematics 2 (2012) 3.

[17] Z. S. Hamidi, S. Chumiran, A. Mohamad, N. Shariff, Z. Ibrahim, N. Radzin, N. Hamzan, N. Anim, A. Alias, American Journal of Modern Physics 2 (2013) 4.

[18] Z.S.Hamidi, Z. Abidin, Z. Ibrahim, N. Shariff, C. Monstein, International Journal of Engineering Research and Development 3 (2012) 3.

[19] Z. S. Hamidi, Z. Abidin, Z. Ibrahim, C. Monstein, N. Shariff, International Journal of Fundamental Physical Sciences 2 (2012) 32-34.

[20] Z. S.Hamidi, et al., International Journal of Fundamental Physical Sciences 2 (2012) 4.

[21] Z. S. Hamidi, N. N. M. Shariff, International Journal of Science and Mathematics 2 (2014) 3.

[22] Z. S. Hamidi, Z. Abidin, Z. Ibrahim, N. Shariff, Indication of radio frequency interference (RFI) sources for solar burst monitoring in Malaysia, ICPAP 2011, AIP Publisher, Indonesia, 2012, pp. 6.

[23] Z. S. Hamidi, N. N. M. Shariff, C. Monstein, The International Journal of Engineering 1 (2012) 3.

[24] Z.S. Hamidi, N. Shariff, Z. Abidin, Z. Ibrahim, C. Monstein, Middle-East Journal of Scientific Research 12 (2012) 6.

[25] Z. S. Hamidi, N. N. M.Shariff, R. Umar, Malaysia Thailand Journal of Physics 3 (2012) 6.

[26] Z. S. Hamidi, N. N. M. Shariff, International Letters of Chemistry, Physics and Astronomy 4 (2014) 29-36.

[27] Z. S. Hamidi, N. N. M. Shariff, M. F. Ali, C. Monstein, W. N. A. W. Zulkifli, M. B. Ibrahim, N. S. Arifin, N. A. Amran, International Letters of Chemistry, Physics and Astronomy 9 (2014) 84-92. 
[28] Z. S. Hamidi, N. N. M. Shariff, C.Monstein, W. N. A. W. Zulkifli, M. B. Ibrahim, N. S. Arifin, N. A. Amran, International Letters of Chemistry, Physics and Astronomy 8 (2014) 13-19.

[29] Z. S. Hamidi, N. N. M. Shariff, C.Monstein, W. N. A. W. Zulkifli, M. B. Ibrahim, N.S. Arifin, N.A. Amran, International Letters of Chemistry, Physics and Astronomy 9 (2014) 8-12.

[30] Z. S. Hamidi, N. N. M. Shariff, International Letters of Chemistry, Physics and Astronomy 7 (2014) 30-36.

[31] Z. S. Hamidi, N. N. M. Shariff, International Letters of Chemistry, Physics and Astronomy 5 (2014) 32-42.

[32] Z. S. Hamidi, N. N. M. Shariff, C. Monstein, Z. A. Ibrahim, International Letters of Chemistry, Physics and Astronomy 7 (2014) 37-44.

[33] Z. S. Hamidi, N. N. M. Shariff, C. Monstein, International Letters of Chemistry, Physics and Astronomy 11(2) (2014) 167-176

[34] Z. S. Hamidi, C. Monstein, N. N. M. Shariff, International Letters of Chemistry, Physics and Astronomy 11(3) (2014) 243-256.

[35] Z. S. Hamidi, N. N. M. Shariff, C. Monstein, W. N. A. Wan Zulkifli, M. B. Ibrahim, N. S. Arifin, N. A. Amran, International Letters of Natural Sciences 5 (2014) 10-17. 